\title{
Mechanism of the Unique Susceptibility of Deep Cortical Glomeruli of Maturing Kidneys to Severe Focal Glomerular Sclerosis ${ }^{1}$
}

\author{
MASAAKI IKOMA, TOSHIMASA YOSHIOKA, IEKUNI ICHIKAWA, AND AGNES FOGO \\ Departments of Pediatrics and Pathology, Vanderbilt University School of Medicine, \\ Nashville, Tennessee 37232-2584
}

\begin{abstract}
The alterations in single glomerular hemodynamics, glomerular size, and development of glomerular sclerosis after subtotal nephrectomy were assessed in $\sim 32-$ d-old young and $>3$-mo-old adult Munich-Wistar rats. In $6 \mathrm{wk}$, young rats developed more pronounced glomerular sclerosis and more marked elevation in blood urea nitrogen. The deep (versus superficial) cortical region of young rats was characterized by having a greater number of glomeruli with advanced sclerosis. Single nephron glomerular filtration rate of superficial glomeruli of the young increased to a much greater extent than whole kidney glomerular filtration rate, whereas there were comparable post-subtotal nephrectomy increases in whole kidney glomerular filtration rate in these two age groups, indicating that the deep glomeruli were exposed to a lesser hemodynamic load than were the superficial. Since the remnant nephrons of young and adult rats achieved equally high glomerular pressures and comparably large glomerular size shortly after subtotal nephrectomy, the unique susceptibility of young glomeruli to sclerosis is attributed to the intrinsic property of these glomeruli, rather than the abnormal hemodynamics or stimuli promoting hypertrophy and mesangial matrix accumulation imposed upon the glomeruli. (Pediatr Res 28: 270-276, 1990)
\end{abstract}

\section{Abbreviations}

BUN, blood urea nitrogen

BW, body weight

GFR, glomerular filtration rate

PAmean, mean planar area of glomeruli

$\mathbf{P}_{\mathrm{GC}}$, glomerular capillary pressure

SBP, systolic blood pressure

SNGFR, single nephron glomerular filtration rate

sNPX, subtotal nephrectomy

In view of the marked functional and structural internephron heterogeneity seen in chronically diseased kidneys in both humans and animals (1-4), various initial immune or nonimmune insults appear to cause loss of a selected population of functioning nephrons. Extensive nephron population loss leads to functional

Received August 29, 1989; accepted April 13, 1990.

Correspondence: Agnes Fogo, M.D., Division of Pediatric Nephrology, Vanderbilt University Medical Center, C-4202 Medical Center North, 21st \& Garland Avenue, Nashville, TN 37232-2584.

Supported by NIH Grant DK 37868. I.I. and A.F. are recipients of the Established Investigatorship Award and Clinician Scientist Award, respectively, from the American Heart Association.

' Portions of these studies were presented at the annual meeting of the American Society of Nephrology, San Antonio, TX, December 11, 1988, and published in abstract form (Kidney Int 35:430, 1989) and metabolic adjustments in remnant intact nephrons. The progression to end-stage kidneys is currently explained by the premise that these alterations are "self-destructive" in nature and induce sclerotic lesions in glomeruli, leading to further reduction in nephron population and perpetuation of the cycle $(4,5)$.

sNPX in animals, which simulates experimentally this reduction in nephron population, indeed leads to glomerular sclerosis closely resembling that in human end-stage kidneys $(3,6)$. In the rat model of SNPX, there are increases in glomerular filtration and glomerular capillary hydraulic pressure in remnant nephrons (3). It was therefore proposed that these hemodynamic changes are one of the important self-destructive mechanisms underlying the subsequent structural damage within the glomerulus $(3,4,7$, 8).

An alternative mechanism has recently been identified on the basis of the ubiquitous observations made in experimental and clinical settings. Hypertrophy of the glomerulus is a phenomenon that is invariably associated with glomerular sclerosis (9-20). When hypertrophic stimuli are imposed on the kidney by unilateral nephrectomy, the glomerular sclerosing process was found to be accelerated in the remnant kidney with experimental glomerulonephritis (9), minimal changes or focal sclerosis (10, 11 ), and diabetic nephropathy (12). On the basis of these and other in vivo and in vitro experiments, it is speculated that alteration in circulating or local hormonal environment as a result of the functioning nephron loss leads to the hypertrophy and extracellular matrix accumulation seen in sclerosing glomeruli $(5,21)$.

Of particular relevance to childhood renal diseases, glomerular sclerosis is often found at autopsy in individuals who underwent unilateral nephrectomy in infancy, in contrast to a lower incidence of focal glomerular sclerosis following nephrectomy in adulthood (22-24). This clinical observation has led to a proposal that remnant glomeruli of maturing kidneys are uniquely susceptible to the development of focal glomerular sclerosis. Unequivocal evidence that the young are indeed predisposed to glomerular damage after nephron loss has recently been obtained from the test in humans of renal function after nephrectomy in childhood versus unilateral renal agenesis by Wikstad et al. (25), and histologic demonstration in experimental animals by Celsi et al. (26) and O'Donnell et al. (27). In contrast to the very good prognosis with normal renal function after loss of a kidney from trauma or transplant donation in the adult, the 15 patients with unilateral renal agenesis studied by Wikstad et al. (25) showed a decline in inulin clearance rates, and nearly half developed microalbuminuria. In light of the above-discussed mechanisms previously tested in a variety of experimental settings, this predisposition of young glomeruli may be attributed to exaggerated hemodynamic abnormality and/or glomerular hypertrophy that follow nephron loss. Alternatively, the glomeruli of the young may be endowed with a unique propensity to develop sclerosis in an accelerated manner at a given hemodynamic load and/or 
in response to stimuli promoting hypertrophy and matrix synthesis. These potential mechanisms for the observed unique susceptibility of the maturing kidney to develop sclerosis were explored in our study.

\section{MATERIALS AND METHODS}

Experimental groups. Experiments were performed in 24 young ( 32 -d-old, $60-90 \mathrm{~g} \mathrm{BW}$, group 1$)$ and 23 adult ( $>3$-moold, 200-240 g BW, group 2) male Munich-Wistar rats. As outlined in Table 1, each of these age groups was further divided into four subgroups.

Group 1a (six young rats) and $2 \mathrm{a}$ (five adult rats) rats were used to evaluate our surgical technique to successfully produce comparable degrees of SNPX in young and adult rats. For this purpose, these animals were randomly picked from the entire pool of 47 rats during the course of our study to estimate the degree of sNPX by observing the magnitude of changes in whole kidney GFR that occurs immediately after sNPX. These rats were anesthetized with Inactin $(100 \mathrm{mg} / \mathrm{kg} \mathrm{BW}$ intraperitoneally, Byk, FRG) and underwent baseline measurements of whole kidney GFR. SNGFR and $\mathrm{P}_{\mathrm{GC}}$ of surface glomeruli were measured in these animals at the time of initial whole kidney GFR measurement to obtain the baseline single nephron hemodynamic patterns, i.e. before sNPX. (Methods of the measurement are detailed below.) After the baseline measurement, rats underwent right nephrectomy and ligation of two or three main branches of the left renal artery by a silk ligature to leave approximately $1 / 6-1 / 8$ total kidney tissue mass, i.e. sNPX. Measurements of whole kidney GFR, SNGFR, and $\mathrm{P}_{\mathrm{GC}}$ were then repeated immediately after $\mathrm{SNPX}$.

In groups $1 \mathrm{~b}(n=6)$ and $2 \mathrm{~b}(n=6)$, whole kidney GFR, SNGFR, and $P_{G C}$ were measured 2 wk after sNPX. These rats were anesthetized with intraperitoneal pentobarbital (25-30 mg/ kg BW; Nembutal, Abbott Laboratories, North Chicago, IL), and SNPX was performed in an identical manner to groups la and $2 \mathrm{a}$. After the recovery from the anesthesia, rats were returned to their cages and maintained on a regular diet for $2 \mathrm{wk}$. At 2 wk, rats were anesthetized with Inactin and the measurements specified above were repeated.

Groups 1c $(n=6)$ and $2 \mathrm{c}(n=6)$ underwent histologic examination at $6 \mathrm{wk}$ after sNPX, which was performed in an identical manner to that described above for group $1 b$ and $2 b$. After sNPX, blood samples were taken periodically over the next 6 wk from tail veins to measure the level of BUN. SBP was also measured before and up to $6 \mathrm{wk}$ after the sNPX using the tail cuff method previously described (28). Six wk after sNPX, remnant kidneys were harvested for histologic evaluation. Both right kidneys, removed at the time of sNPX, and left remnant kidneys, obtained $6 \mathrm{wk}$ later, were fixed in $10 \%$ neutral buffered formalin and processed for evaluation of glomerular sclerosis and glomerular size as detailed below.
To serve as time controls, group $1 \mathrm{~d}(n=6)$ and $2 \mathrm{~d}(n=6)$ rats underwent sham operation, and were subsequently maintained, like the other groups, on a regular diet for $6 \mathrm{wk}$ before sacrifice.

Micropuncture measurements. In groups $1 \mathrm{a}, 1 \mathrm{~b}, 2 \mathrm{a}$, and $2 \mathrm{~b}$, micropuncture measurements were performed as follows. After anesthesia, an indwelling polyethylene catheter (PE-50, Clay Adams, Parsippany, NJ) was placed into the left jugular vein for infusion of plasma and inulin. The left femoral artery was catheterized to monitor mean systemic arterial pressure using an electronic transducer (model p23Db, Gould Inc., Cleveland, $\mathrm{OH}$ ) that was connected to a recorder (model $2200 \mathrm{~S}$, Gould Inc.). The left ureter was cannulated with PE-10 tubing for subsequent collection of urine. The left kidney was suspended on a Lucite holder, its surface illuminated with a fiberoptic light source, and bathed with $0.9 \% \mathrm{NaCl}$. Volume losses due to surgical preparation were replaced with plasma, infused in a volume equal to $1 \%$ of BW over $30 \mathrm{~min}$, followed by a maintenance infusion at the rate of $1.2 \mathrm{~mL} / \mathrm{h}(29)$. After a priming dose $(0.5 \mathrm{~mL})$ of $0.9 \%$ $\mathrm{NaCl}$ containing $5 \%$ inulin, a maintenance infusion was started and continued throughout each experiment at the rate of 1.2 $\mathrm{mL} / \mathrm{h}$.

Timed (1-2 $\mathrm{min}$ ) samples of fluid were collected from the surface proximal tubules from two or three nephrons for determination of flow rate, inulin concentration, and calculation of tubule-to-plasma inulin concentration ratio, hence, SNGFR. The rate of fluid collection was adjusted to maintain a column of polymer oil, three to four tubule diameters in length, in a constant position just distal to the site of puncture. In conjunction with the tubule fluid collections, femoral arterial blood samples were also obtained for the determination of hematocrit and plasma inulin concentration. Urine was collected from the left ureters for determination of urine flow rate and inulin concentration, which were used for the calculation of whole kidney GFR.

Hydraulic pressures were monitored in accessible surface glomeruli with a continuous-recording servo-null micropipette transducer system (model 3, Instrumentation for Physiology and Medicine, San Diego, CA). Micropipettes with outer tip diameter of 1-2 $\mu \mathrm{m}$ containing $2.0 \mathrm{M} \mathrm{NaCl}$ were used. Hydraulic output from the servo-null system was converted electronically to a recorder (model 2200S, Gould Inc.) by means of a pressure transducer.

Histological study. Sections of $3-\mu \mathrm{m}$ thickness were made, stained with periodic acid-Schiff, and examined microscopically (BH2, Olympus Optical Co., Ltd., Tokyo, Japan).

PAmean was determined for young and adult glomeruli on sections of baseline control kidney removed at sNPX (groups 1d and $2 \mathrm{~d}$ ) and the remnant kidney sections (groups $1 \mathrm{c}$ and $2 \mathrm{c}$ ). The planar area of each glomerulus was determined on these sections using a computerized planimeter (Micro-plan II, Monsanto Corp., Natick, MA). The values of these planar areas,

Table 1. Summary of experimental protocols*

\begin{tabular}{|c|c|c|c|}
\hline Experimental group & Surgery & Time of study & Measurements \\
\hline \multicolumn{4}{|l|}{ Young rats } \\
\hline Group la $(n=6)$ & sNPX & At the time of surgery & Function 1 \\
\hline Group $1 \mathrm{~b}(n=6)$ & sNPX & 2 wk after surgery & Function 2 \\
\hline Group Ic $(n=6)$ & sNPX & $0-6$ wk after surgery & Function $3 \&$ histology \\
\hline Group 1d $(n=6)$ & Sham operation & $0-6$ wk after surgery & Function 3 \\
\hline \multicolumn{4}{|l|}{ Adult rats } \\
\hline Group $2 \mathrm{a}(n=5)$ & sNPX & At the time of surgery & Function 1 \\
\hline Group $2 b(n=6)$ & sNPX & 2 wk after surgery & Function 2 \\
\hline Group 2c $(n=6)$ & sNPX & $0-6$ wk after surgery & Function $3 \&$ histology \\
\hline Group $2 \mathrm{~d}(n=6)$ & Sham operation & $0-6$ wk after surgery & Function 3 \\
\hline
\end{tabular}

* Function 1: whole kidney GFR, SNGFR, and $\mathrm{P}_{\mathrm{GC}}$ before and immediately àfter sNPX; function 2: whole kidney GFR, SNGFR, and $\mathrm{P}_{\mathrm{GC}}$; function 3: periodical measurements of BW, SBP, and BUN over the 6-wk study period. Histology includes evaluation of sclerosis index and PAmean in removed kidneys (obtained at the time of sNPX to serve as baseline controls) and remnant kidney (6 wk after sNPX). 


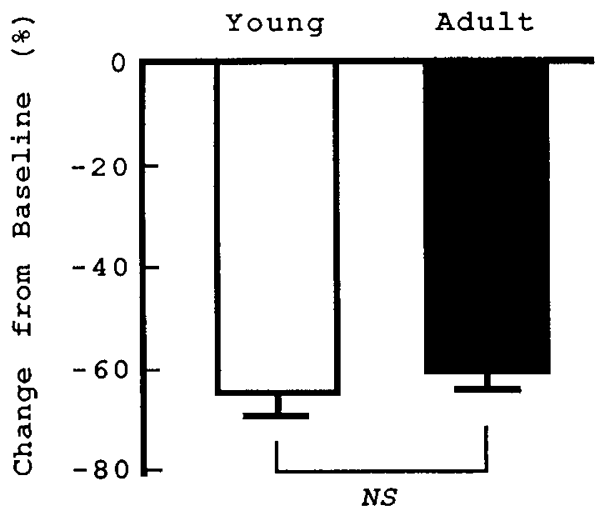

Fig. 1. Changes in whole kidney GFR per $100 \mathrm{~g}$ BW expressed as percentages of baseline values in group 1a young and group $2 \mathrm{a}$ adult animals. These values represent the differences between values obtained immediately before and immediately after SNPX (mean $\pm 1 \mathrm{SE}$ ).
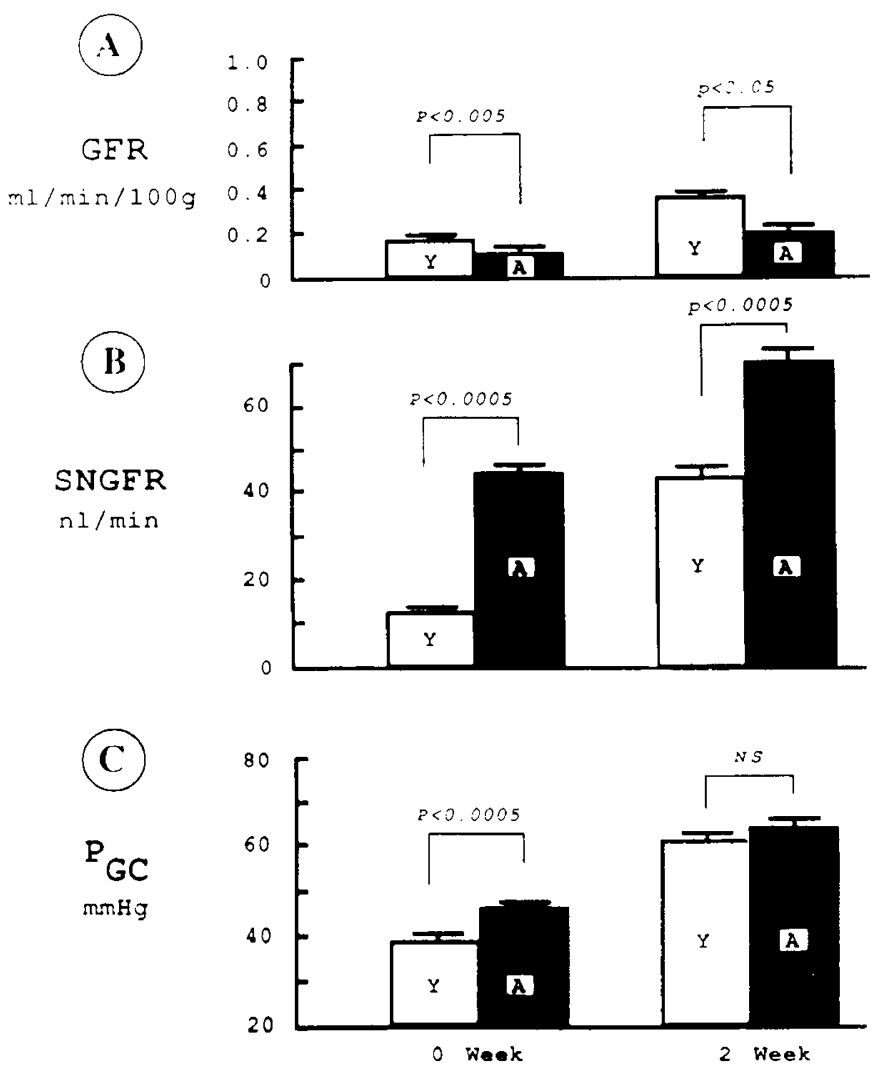

Fig. 2. Values for whole kidney GFR per $100 \mathrm{~g}$ BW $(A)$, superficial SNGFR $(B)$, and $\mathrm{P}_{\mathrm{GC}}(C)$ in young $(Y)$ and adult $(A)$ animals assessed at the time of sNPX ( 0 Week, groups $1 \mathrm{a}$ and $2 \mathrm{a}$ ) and $2 \mathrm{wk}$ after sNPX ( 2 Weeks, groups $1 \mathrm{~b}$ and $2 \mathrm{~b}$ ). Whole kidney GFR and SNGFR values at 0 Week represent those immediately after sNPX, and $\mathrm{P}_{\mathrm{GC}}$ values at 0 Week represent those immediately before $\mathrm{SNPX}$ (mean $\pm 1 \mathrm{SE})$.

measured in 50-60 glomeruli, were averaged for each thinsection specimen, giving the PAmean.

In addition to this determination for the entire glomerular population, the planar area was assessed separately for superficial and deep cortical glomeruli. Superficial glomeruli were defined as the glomeruli located within three glomerular-diameter lengths of the surface of the cortex. Deep glomeruli were defined as the total glomeruli excluding the superficial glomeruli, i.e. glomeruli located more than three glomerular-diameter lengths from the surface.

Glomerular sclerosis was semiquantitated at the whole kidney level in control and remnant kidney sections using the semi- quantitative score (sclerosis index, grade 0-4) of Raij et al. (30), where grade 1 represents involvement of up to $25 \%$ of glomerulus, whereas grade 4 represents sclerosis of $75-100 \%$ of the glomerulus. The whole kidney sclerosis index was obtained by averaging scores for all glomeruli on one or two thin coronal sections, containing a minimum of 100 glomeruli.

The degree of sNPX was compared in separate groups of young $(n=5)$ and adult animals $(n=5)$ at 2 wk after sNPX by calculating the number of remnant glomeruli. The relative proportion of total renal parenchyma versus glomerular area was measured morphometrically with a computerized digitizing planimeter by assessing total glomerular area and total renal area on a cross-section of the remnant kidney. Mean glomerular tuft volume $\left(\mathrm{GV}_{\mathrm{T}}\right)$ was calculated from the mean glomerular tuft area $\left(A_{G}\right)$ by the formula:

$$
\text { Mean } \mathrm{GV}_{\mathrm{T}}=\beta / k \times\left(\mathrm{A}_{\mathrm{G}}\right)^{3 / 2}
$$

where $\beta=1.38$, the shape coefficient for spheres, which is the idealized glomerular tuft shape, and $k=1.1$, a size distribution coefficient $(31,32)$. The volume of the remnant kidney was measured before fixation and multiplied by the coefficient for glomerular density, the ratio of total renal parenchymal area to glomerular area to arrive at total glomerular volume. The number of glomeruli per remnant kidney was then calculated by dividing total glomerular volume by mean glomerular volume.

Analytic. BUN was measured using a BUN analyzer (BUN Analyzer II, Beckman Instrument, Inc., Fullerton, CA). The volume of fluid collected from individual proximal tubules by micropuncture was estimated from the length of fluid column in a constant-bore capillary tube of known internal diameter. Inulin concentration in plasma and urine was determined by the macroanthrone method (33). Inulin concentration in tubule fluid was determined by the method of Vurek and Pegram (34).

Statistical analysis. Results were compared by paired or unpaired $t$ test, as appropriate, and deemed to have statistical significance when $p<0.05$.

\section{RESULTS}

Validation of comparable degree of $S N P X$ in young versus adult rats. Changes in whole kidney GFR/100 g BW achieved immediately after sNPX in young (group $1 \mathrm{a}$ ) and adult (group $1 \mathrm{~b}$ ) rats are illustrated in Figure 1. Whole kidney GFR thus corrected for animal weight fell, on average, from $0.51 \pm 0.03$ at baseline to $0.18 \pm 0.02 \mathrm{~mL} / \mathrm{min} / 100 \mathrm{~g} \mathrm{BW}$ per left kidney immediately after sNPX in young rats. Similarly, in adult rats, the corrected GFR fell from $0.30 \pm 0.01$ at baseline to $0.11 \pm 0.01 \mathrm{~mL} / \mathrm{min} /$ $100 \mathrm{~g} \mathrm{BW}$ immediately after sNPX. Thus, there was the same degree of change, $\sim 60 \%$ reduction in GFR in both groups $(p<$ 0.0005 , baseline versus post-sNPX). Moreover, the number of remaining glomeruli per kidney was comparable in young and adult animals killed at $2 \mathrm{wk}$ after sNPX, on average $7991 \pm 820$ and $8561 \pm 1229$, respectively $(p>0.4$ between young and adults). Because these animals were randomly picked from the entire pool of animals during the course of our study, these data are taken to indicate that comparable sNPX was achieved in the young and adult rats of other experimental groups as well.

Changes in whole kidney and single nephron glomerular hemodynamics. Values for whole kidney GFR/100 g BW averaged $0.38 \pm 0.07 \mathrm{~mL} / \mathrm{min} / 100 \mathrm{~g} \mathrm{BW}$ in group $1 \mathrm{~b}$ young animals $(2$ wk after sNPX), a value $\sim 111 \%$ higher than that measured immediately after sNPX in group la young rats (Fig. $2 A$ ). Likewise, values for whole kidney GFR averaged $0.20 \pm 0.01$ $\mathrm{mL} / \mathrm{min} / 100 \mathrm{~g} \mathrm{BW}$ in group $2 \mathrm{~b}$ adult animals ( $2 \mathrm{wk}$ after $\mathrm{sNPX}$ ), a value $82 \%$ higher than that measured immediately after sNPX in group $2 \mathrm{a}$ adult rats (Fig. $2 A$ ). Thus, after the comparable reduction found immediately after SNPX discussed above, whole kidney GFR increased in 2 wk in both young and adult rats to a comparable degree.

In contrast to this comparable pattern seen in whole kidney 
GLOMERULAR SCLEROSIS OF MATURING KIDNEYS

Table 2. Systemic parameters measured in group $1 c, 1 d, 2 c$, and $2 d$ awake animals*

\begin{tabular}{lcccccccccc}
\hline & \multicolumn{4}{c}{ BW (g) } & \multicolumn{1}{c}{ BUN (mg/dL) } & \multicolumn{1}{c}{ SBP (mm Hg) } \\
\hline Wk & 0 & 2 & 4 & 6 & 0 & 2 & 4 & 6 & 0 & 6 \\
Young rats & & & & & & & & & \\
$\quad$ Group 1c (sNPX) & $74 \pm 2$ & $84 \pm 7 \dagger$ & $116 \pm 7 \dagger$ & $149 \pm 9$ & & $40 \pm 4 \dagger$ & $46 \pm 4 \dagger$ & $74 \pm 11 \dagger$ & $101 \pm 4$ & $153 \pm 12 \dagger$ \\
$\quad$ Group 1d (cont) & $77 \pm 3$ & $128 \pm 9$ & $155 \pm 6$ & $211 \pm 7$ & $17 \pm 1$ & $15 \pm 1$ & $15 \pm 1$ & $17 \pm 1$ & $100 \pm 5$ & $116 \pm 4$ \\
Adult Rats & & & & & & & & & & \\
$\quad$ Group 2c (sNPX) & $229 \pm 4$ & $198 \pm 7 \dagger$ & $213 \pm 15 \dagger$ & $244 \pm 6 \dagger$ & & $49 \pm 3 \dagger$ & $50 \pm 3 \dagger$ & $58 \pm 8 \dagger$ & $115 \pm 5$ & $177 \pm 9 \dagger$ \\
$\quad$ Group 2d (cont) & $228 \pm 4$ & $230 \pm 6$ & $266 \pm 6$ & $295 \pm 15$ & $19 \pm 2$ & $18 \pm 1$ & $20 \pm 1$ & $17 \pm 5$ & $120 \pm 1$ & $123 \pm 7$ \\
\hline
\end{tabular}

$*$ Values are given as mean \pm SEM.

$\dagger p<0.05$, sNPX rats $v s$ control sham-operated rats (cont).

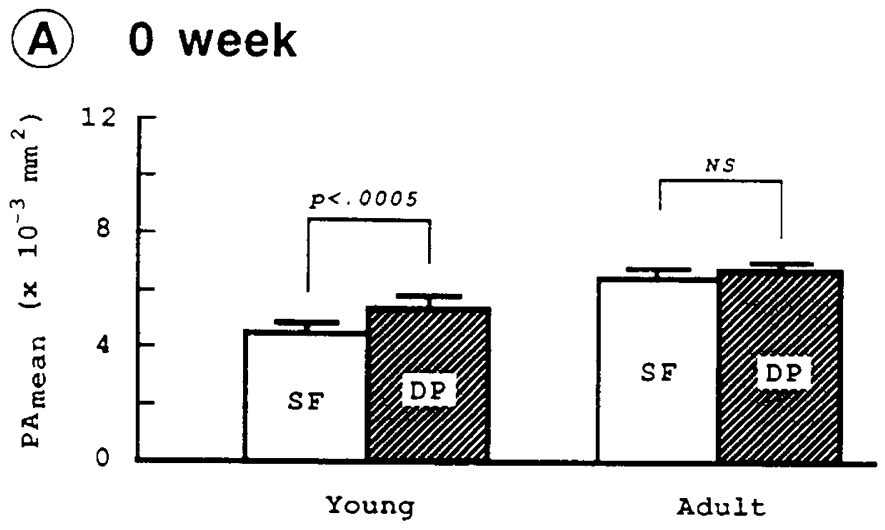

\section{(B) 6 week}

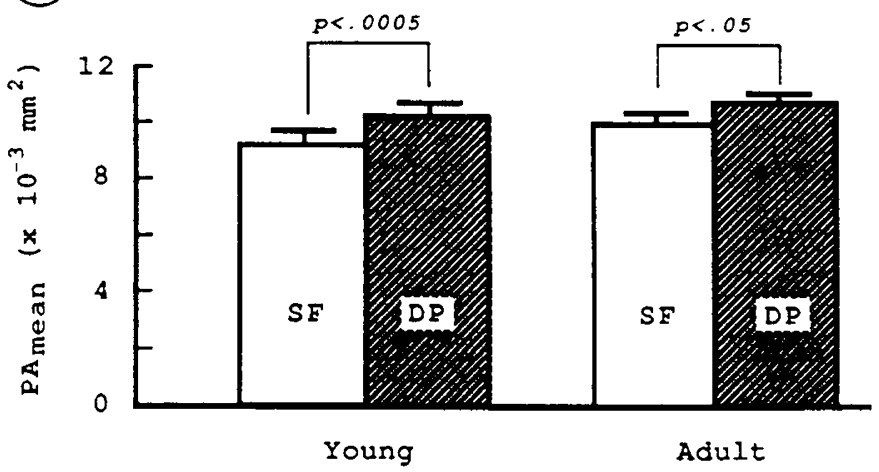

Fig. 3. PAmean determined on thin-section specimens from nephrectomized right kidneys at the time of $\operatorname{sPX}$ ( $A$, group 1a young and group $2 \mathrm{a}$ adult rats) and remnant left kidneys at the time of sacrifice $6 \mathrm{wk}$ after $\operatorname{sNPX}(B$, group $1 c$ young and group $2 c$ adult rats). Values from superficial $(S F)$ and deep cortical $(D P)$ glomeruli are given separately (mean $\pm 1 \mathrm{SE})$.

GFR changes, a marked difference was seen at the single nephron level between young and adult rats at $2 \mathrm{wk}$ after sNPX. Thus, values for SNGFR averaged $44.3 \pm 3.1 \mathrm{~nL} / \mathrm{min}$ in group $1 \mathrm{~b}$ young animals ( 2 wk after sNPX), a value $\sim 240 \%$ above that measured immediately after SNPX in group la young rats (13.1 $\pm 0.9 \mathrm{~nL} / \mathrm{min}$ ), whereas in adult rats, SNGFR values averaged $70.9 \pm 3.0 \mathrm{~nL} / \mathrm{min} 2 \mathrm{wk}$ after sNPX (group $2 \mathrm{~b}$ ), a value only $\sim 60 \%$ above that measured immediately after sNPX (group $1 \mathrm{~b}$, $45.3 \pm 2.9 \mathrm{~nL} / \mathrm{min}, p<0.005$ for the difference in percentage) (Fig. $2 B$ ). Similarly, young rats had a more marked increase in $\mathrm{P}_{\mathrm{GC}}$ than the adult rats. Thus, values for $\mathrm{P}_{\mathrm{GC}}$ averaged $61 \pm 2$ $\mathrm{mm} \mathrm{Hg}$ in group $1 \mathrm{~b}$ young animals ( 2 wk after sNPX), a value $23 \mathrm{~mm} \mathrm{Hg}$ above that measured immediately before sNPX in group la young rats $(38 \pm 1 \mathrm{~mm} \mathrm{Hg})$. In adult rats, $\mathrm{P}_{\mathrm{GC}}$ values averaged $65 \pm 3 \mathrm{~mm} \mathrm{Hg} 2$ wk after sNPX (group 2b), a value only $19 \mathrm{~mm} \mathrm{Hg}$ above that measured immediately before sNPX

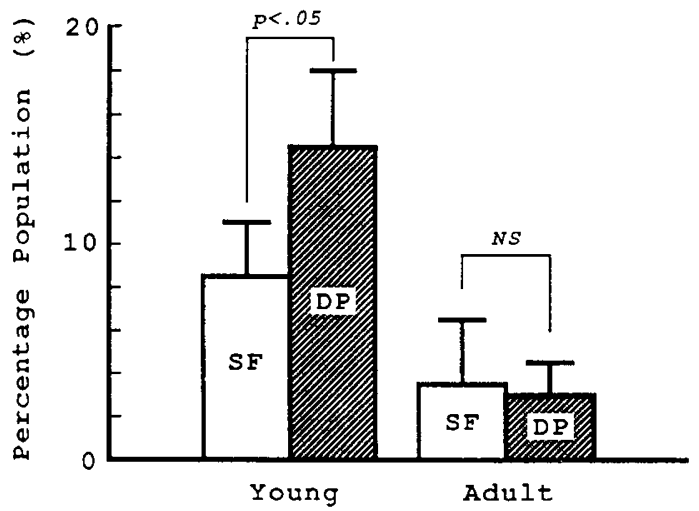

Fig. 4. Percentage population of glomeruli that had sclerosis index (0-4 scale) greater than 3 in remnant left kidneys from group 1c young and group $2 \mathrm{c}$ adult animals examined $6 \mathrm{wk}$ after sNPX. Values from superficial $(S F)$ and deep cortical $(D P)$ glomeruli are given separately $($ mean $\pm \mathrm{SE})$.

(group 2a, $46 \pm 1 \mathrm{~mm} \mathrm{Hg}, p<0.025$ for the difference in changes in young versus adult) (Fig. $2 C$ ), resulting in levels comparable to those achieved in young rats.

Six-wk post-sNPX course in young and adult rats. Mean values for BW, BUN, and SBP measured in group 1c young and group $2 \mathrm{c}$ adult rats at the onset of study and at subsequent pertinent time intervals are shown in Table 2. In young rats, BW on the day of sNPX was essentially identical in group 1c nephrectomized and group $1 \mathrm{~d}$ control rats, averaging $72 \pm 2$ versus $77 \pm$ $3 \mathrm{~g}$, respectively. BW of the young rats $6 \mathrm{wk}$ after sNPX, however, was significantly and substantially lower than that of their controls, averaging $149 \pm 9$ versus $211 \pm 7 \mathrm{~g}(p<0.0005)$. Essentially identical patterns were seen in group $2 \mathrm{c}$ adult rats, as shown in Table 2. As also shown in Table 2, BUN values were elevated over the control values throughout the 2 - to 6 -wk period in both young and adult rats. Whereas the elevated BUN level remained relatively constant in group $2 \mathrm{c}$ adult rats during the 2- to 6-wk period, BUN increased progressively in group 1c young rats. By $6 \mathrm{wk}, \mathrm{BUN}$ levels were significantly and substantially higher $(p$ $<0.005$ ) than those measured in group $2 c$ rats after an identical post-sNPX period. Given the comparable degree of the compromise in whole kidney GFR achieved in post-sNPX discussed above in young and adult rats, this progressive rise in BUN seen only in the young appears to reflect their maturational increase in BUN production, which was not accompanied by otherwiseexpected parallel maturational increase in whole kidney GFR. Finally, as also seen in Table 2, both animals achieved typical and comparable post-sNPX increase in SBP.

Glomerular histology 6 wk after $s N P X$. Figure $3 A$ summarizes values for PAmean determined in the removed right kidney at the time of sNPX (groups 1a and 2a). Not surprisingly, baseline values for PAmean were significantly and substantially smaller for both superficial and deep glomeruli in the young than the adult, on average $4.50 \pm 0.15$ and $5.20 \pm 0.1510^{-3} \mathrm{~mm}^{2}$, respectively, versus $6.83 \pm 0.34(p<0.0005)$ and $6.99 \pm 0.19$ $10^{-3} \mathrm{~mm}^{2}(p<0.0005$, young versus adult), respectively. Of 


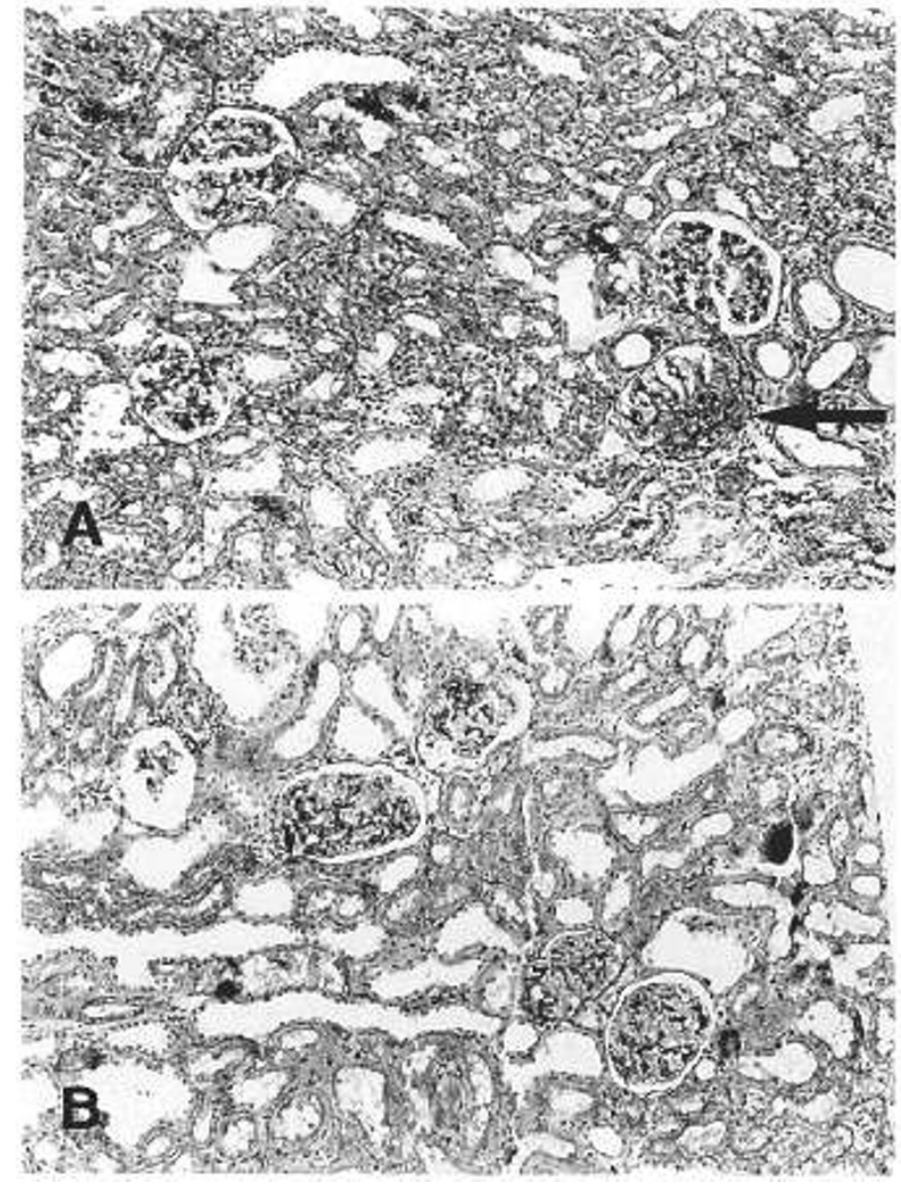

Fig. 5. Glomerular histology at $6 \mathrm{wk}$ after sNPX in young rats $(A)$ and adult rats $(B)$. Deeper nephrons (arrow) show more severe lesions than superficial nephrons in the young rats. The lesions are more focal and also more severe than in adult $(B)$. The glomeruli have achieved comparable absolute size (periodic acid Schiff, $\times 60$ ).

note, within the normal baseline kidney of the young, but not the normal baseline of the adult, a significant difference in PAmean $(p<0.0005)$ between superficial and deep glomeruli was noted (Fig. $3 A$ ). This heterogeneity in glomerular size within the kidney was still present in young rats at $6 \mathrm{wk}$ after sNPX (Fig. $3 B$ ). Additionally, deep glomeruli in sNPX adults increased $\sim 60 \%$ in size versus only $\sim 47 \%$ increase in superficial glomeruli, resulting in development of significant size heterogeneity within individual kidneys in these rats. Thus, PAmean averaged $9.59 \pm$ 0.33 ( $113 \%$ increase) and $10.94 \pm 0.1910^{-3} \mathrm{~mm}^{2}$ (110\% increase) in superficial and deep glomeruli, respectively, in group 1c young rats ( 6 wk post-sNPX); and $10.03 \pm 0.73$ (47\% increase) and $11.16 \pm 0.2510^{-3} \mathrm{~mm}^{2}$ (60\% increase) in superficial and deep glomeruli, respectively, in group $2 \mathrm{c}$ adult rats ( $6 \mathrm{wk}$ post-sNPX). Six wk after sNPX, PAmean of both superficial and deep glomeruli from young rats were comparable to those of corresponding superficial and deep glomeruli from adult rats (young superficial versus adult superficial, NS, young deep versus adult deep, NS).

Values for whole kidney sclerosis index in group 1c young rats (6 wk after sNPX) were significantly and markedly higher than those of group $2 c$ adult rats, averaging $0.69 \pm 0.15$ versus 0.26 \pm 0.07 , respectively $(p<0.025)$. Figure 4 shows sclerosis index values determined separately for superficial and deep cortical glomeruli. As shown in Figure 4, a larger population of glomeruli was found to have advanced sclerosis (sclerosis index $>3.0$ ) in the deep cortex of young rats, compared with the superficial glomeruli of the same kidney (deep $14.3 \pm 4.1$ versus superficial $8.5 \pm 2.5 \%, p<0.05$ ), whereas such a pattern of severe sclerosis distribution within individual kidneys was not found in adult kidneys (deep $3.2 \pm 1.2$ versus superficial $3.7 \pm 2.8 \%$, NS). These histologic findings in young and adult animals at 6 wk after sNPX are illustrated in Figure 5.

\section{DISCUSSION}

Our observations for $6 \mathrm{wk}$ after subtotal nephrectomy in rats are remarkably similar to the findings made at the whole kidney level by Celsi et al. (26) in unilaterally nephrectomized rats monitored over a 6-mo period. In their study, whole kidney GFR per BW progressively decreased over the 6-mo post-sNPX period in the young, contrasting to adult GFR values, which leveled off by 2 mo. Similarly, in our study, whole kidney GFR per BW of young rats, as reflected by increasing BUN, decreased progressively throughout the 6-wk post-sNPX period, as opposed to remarkably stable adult BUN values during the 2 - to 6 -wk period (Table 2). Also in agreement with their observations, we found that the remnant glomeruli of young animals examined at the completion of study were characterized by more severe sclerosis than those of adult animals. Thus, the functional and histologic processes of remnant kidneys observed in their studies were duplicated in our study but in an accelerated manner, a pattern reminiscent of the familiar notion that the rate of progression of sclerosis in remnant glomeruli has a direct relationship with the degree of initial nephron loss.

By investigating the function and morphology at the single glomerular level, we have made additional interesting observations. At baseline, only young Munich-Wistar rats show nephron size heterogeneity of superficial versus deep cortical glomeruli, which is commonly seen in human kidneys. Glomerular size heterogeneity in most mammals decreases with centrifugal maturation, and varies among species (35). Moreover, the higher incidence of advanced glomerular sclerosis in deep than in superficial nephrons often seen in humans, which has heretofore not been noted in the vast volume of studies conducted in the adult animal models of SNPX, was duplicated in our young sNPX Munich-Wistar rats (Fig. 4). A similar heterogeneity within the kidney was also noted at the functional level in a more pronounced fashion in young animals. Thus, although whole kidney GFR per BW increased to comparable degree (approximately 2 -fold) during the 2 -wk post-sNPX period in the two age groups, SNGFR increased to a much greater extent in the young than in the adult animals (i.e. by $\sim 240$ versus $\sim 60 \%$ ). It is of note that the SNGFR values were determined on superficial nephrons. Thus, the increase in whole kidney GFR must have been accomplished primarily by rises in the filtration rate of superficial nephrons, and remnant deep nephrons' filtration rate may, in fact, have fallen markedly in young animals. ${ }^{2} \mathrm{~A}$ similar difference in single GFR between young and adult animals was reported by Aschinberg et al. (37). Whereas an increase in single glomerular perfusion rates was confined to the deeper nephrons in adults, superficial nephrons contributed most to the adaptive increase in whole kidney GFR in young animals (37). Given that the rise in SNGFR typical for the remnant nephrons after nephrectomy is achieved solely by the rises in glomerular pressure and glomerular plasma flow rate $(3,38)$, the more severe glomerular sclerosis subsequently noted in deeper glomeruli speaks against the currently prevailing assumption that the predisposition of deeper glomeruli to the development of severe sclerosis is attributable to a more pronounced hemodynamic load in these glomeruli (4).

More severe sclerosis was noted not only in deep but also in superficial glomeruli when young and adult remnant kidneys were compared (Fig. 4). Of the factors potentially influential on the progression of glomerular sclerosis $(4,5)$, we assessed two in our study. Measurements of $P_{G C}$ in superficial nephrons dem-

${ }^{2}$ The more pronounced increase in SNGFR of superficial nephrons observed in our study is consistent with the notion that the young animals examined in our study are at the final stage of maturational centrifugal growth (35-37); that is, the increase in whole kidney function is accomplished primarily by maturational increase in size and function of superficial nephrons. 
onstrated that $\mathrm{P}_{\mathrm{GC}}$ increased to comparably high levels $(\sim 60 \mathrm{~mm}$ $\mathrm{Hg}$ ) in young and adult animals within $2 \mathrm{wk}$ after sNPX. The size of glomeruli achieved in remnant nephrons was also comparable between the two age groups. In a previous study, a tight link was found between glomerular hypertrophy and sclerosis (39). In this study, young rats underwent more marked acceleration of glomerular hypertrophy than adults, thus achieving the same absolute glomerular size after sNPX. However, the percent increase in size after sNPX in young rats was similar in superficial versus deep glomeruli, suggesting an existence of mechanisms other than abnormal accelerated hypertrophy underlying the more severe sclerosis of deep glomeruli.

In addition to the above specific information regarding the uniqueness of maturing glomeruli, the results from our comparative study have broadened our view in interpreting the data from various experimental studies that are designed to investigate the pathophysiologic mechanisms underlying the progression of glomerular disease. The readers may recall that the experimental strategy entertained by investigators in the field often includes demonstration of the effects of various experimental maneuvers, drugs, dietary regimens, etc. on the progression of glomerular sclerosis without affecting candidate pathophysiologic mechanisms. Such data were extrapolated into a general scheme of pathophysiology applied to all forms of glomerular sclerosis by taking data as evidence against the importance of such mechanisms in question (40-44). In this regard, our data showing the unique susceptibility of young remnant glomeruli to sclerosis despite glomerular pressure and size comparable to or even below adult levels point to the significance of host-dependent factors as an important determinant for the progression of glomerular sclerosis, which may potentially be altered by these experimental maneuvers. ${ }^{3}$ Of note, the notion has been widely accepted in recent years that local or systemic growth factors that promote glomerular hypertrophy may underlie the development and progression of glomerular sclerosis. Numerous known growth factors have been examined and found to have an effect on matrix production by mesangial cells in culture (21). Most recently, Doi et al. (18) have documented in vivo that overexpression of growth hormone is directly linked to glomerular hypertrophy and sclerosis. It is conceivable then, given that the normal immature glomeruli are already in a milieu comprised of abundant known and unknown growth factors, that with growth and development in this milieu occurring in a centrifugal fashion, superimposition of additional growth promoters after experimental reduction in nephron population may contribute in part to the unique susceptibility of these glomeruli to sclerosis.

Acknowledgments. The authors thank Teresa Bills for technical assistance and Mary Beehan for secretarial assistance.

\footnotetext{
${ }^{3}$ Recently, attempts have been made to explain the dissociation between the degree of increase in glomerular pressure and that of progression of glomerular sclerosis often seen in various experimental settings by invoking Laplace's law, i.e. the potentially injurious effect of increased wall tension imposed upon the glomerular capillary wall is a product not only of the level of glomerular capillary pressure but also of the diameter of the capillary lumen, which is likely to be altered in hypertrophic glomeruli (45). Our observation of marked difference in sclerosis between the two age groups cannot be explained on the basis of this concept inasmuch as this product was comparable in young and adult remnant glomeruli.
}

\section{REFERENCES}

1. Ichikawa I, Hoyer JR, Seiler MW, Brenner BM 1982 Mechanism of glomerulotubular balance in the setting of heterogeneous glomerular injury. $\mathrm{J}$ Clin Invest 69:185-198

2. Kramp RA, MacDowell M, Gottschalk CW, Oliver JR 1974 A study by microdissection and micropuncture of the structure and the function of the kidneys and the nephrons of rats with chronic renal damage. Kidney Int 5:147-176

3. Hostetter TH, Olson JL, Rennke HG, Venkatachalam MA, Brenner BM 1981 Hyperfiltration in remnant nephrons: a potentially adverse response to renal ablation. Am J Physiol 241:F85-F93

4. Brenner BM, Meyer TW, Hostetter TH 1982 Dietary protein intake and the progressive nature of kidney disease: the role of hemodynamically mediated glomerular injury in the pathogenesis of progressive glomerular sclerosis in aging, renal ablation, and intrinsic renal disease. N Engl J Med 307:652-659
5. Klahr S, Schreiner G, Ichikawa I 1988 The progression of renal disease. N Engl J Med 318:1657-1666

6. Purkerson ML, Hoffsten PE, Klahr S 1976 Pathogenesis of the glomerulopathy associated with renal infarction in rats. Kidney Int 9:407-417

7. Hostetter TH, Meyer TW, Rennke HG, Brenner BM 1986 Chronic effects of dietary protein in the rat with intact and reduced renal mass. Kidney Int 30:509-517

8. Anderson S, Diamond JR, Karnovsky ML, Brenner BM 1988 Mechanisms underlying transition from acute glomerular injury to late glomerular sclerosis in a rat model of nephrotic syndrome. J Clin Invest 82:1757-1768

9. Teodoru CV, Saifer A, Frankel H 1959 Conditioning factors influencing evolution of experimental glomerulonephritis in rabbits. Am J Physiol 196:457-460

10. Glasser RJ, Velosa JA, Michael AF 1977 Experimental model of focal sclerosis I. Relationship to protein excretion in aminonucleoside nephrosis. Lab Invest 36:519-526

11. Fries JWU, Sandstrom DJ, Meyer TW, Rennke HG 1989 Glomerular hypertrophy and epithelial cell injury modulate progressive glomerulosclerosis in the rat. Lab Invest 60:205-218

12. Steffes MW, Brown DM, Mauer SM 1978 Diabetic glomerulopathy following unilateral nephrectomy in the rat. Diabetes 27:35-41

13. Seyer-Hansen K, Hansen J, Gundersen HJG 1980 Renal hypertrophy in experimental diabetes. Diabetologia 18:501-505

14. Neugarten J, Feiner HD, Schacht RG, Gallo GR, Baldwin DS 1982 Aggravation of experimental glomerulonephritis by superimposed clip hypertension. Kidney Int 22:257-263

15. Ichikawa I, Purkerson ML, Klahr S, Troy JL, Martinez-Maldonado M, Brenner BM 1980 Mechanism of reduced glomerular filtration rate in chronic malnutrition. J Clin Invest 65:982-988

16. Halliburton IW, Thomson RY 1965 Chemical aspects of compensatory renal hypertrophy. Cancer Res 25:1882-1887

17. Yoshida Y, Fogo A, Ichikawa I 1989 Glomerular hemodynamic changes vs hypertrophy in experimental glomerular sclerosis. Kidney Int 35:654-660

18. Doi T, Striker LJ, Quaife C, Conti FG, Palmiter R, Behringer R, Brinster R, Striker GE 1988 Progressive glomerulosclerosis develops in transgenic mice chronically expressing growth hormone and growth hormone releasing factor but not in those expressing insulinlike growth factor-1. Am J Path 131:398403

19. Fogo A, Hawkins EP, Berry PL, Glick AD Chiang ML, MacDonell Jr RC Ichikawa I 1990 Glomerular hypertrophy in minimal change disease predicts subsequent progression to focal glomerular sclerosis. Kidney Int 38:115-123

20. El-Khatib MT, Becker GJ, Kincaid-Smith PS 1987 Morphometric aspects of reflux nephropathy. Kidney Int 32:261-266

21. Fogo A, Ichikawa I 1989 Evidence for the central role of glomerular growth promoters in the development of sclerosis. Semin Nephrol 9:329-342

22. Kiprov DD, Colvin RB, McClusky RT 1982 Focal and segmental glomerulosclerosis and proteinuria associated with unilateral renal agenesis. Lab Invest 46:275-281

23. Thorner PS, Arbus GS, Celermajer DS, Baumal R 1984 Focal segmental glomerulosclerosis and progressive renal failure associated with a unilateral kidney. Pediatrics 73:806-810

24. Bhathena DB, Julian BA, McMorrow RG, Baehler RW 1985 Focal sclerosis of hypertrophied glomeruli in solitary functioning kidneys of humans. Am J Kidney Dis 5:226-232

25. Wikstad I, Celsi G, Larsson L, Herin P, Aperia A 1988 Kidney function in adults born with unilateral renal agenesis or nephrectomized in childhood. Pediatr Nephrol 2:177-182

26. Celsi G, Bohman S-O, Aperia A 1987 Development of focal glomerulosclerosis after unilateral nephrectomy in infant rats. Pediatr Nephrol 1:290-296

27. O'Donnell MP, Kasiske BL, Raij L, Keane WF 1985 Age is a determinant of the glomerular morphologic and functional responses to chronic nephron loss. J Lab Clin Med 106:308-313

28. Pfeffer JM, Pfeffer MA, Frohlich ED 1971 Validity of an indirect tail-cuff method for determining systolic arterial pressure in unanesthetized normotensive and spontaneously hypertensive rats. J Lab Clin Med 78:957-962

29. Ichikawa I, Maddox DA, Cogan MG, Brenner GM 1978 Dynamics of glomerular ultrafiltration in euvolemic Munich-Wistar rats. Renal Physiol 1:121131

30. Raij L, Azar S, Keane W 1984 Mesangial immune injury, hypertension, and progressive glomerular damage in Dahl rats. Kidney Int 26:137-143

31. Beukers JJB, van der Wal A, Hoedemaker PJ, Weening JJ 1987 Converting enzyme inhibition and progressive glomerulosclerosis in the rat. Kidney Int 32:794-800

32. Weibel ER 1979 Stereological Methods: Practical Methods for Biological Morphometry. Academic Press, Inc, London, pp 51-57

33. Führ J, Kazmarczyk J, Krüttgen CD 1955 Eine einfache colorimetrische Methode zur Inulinbestimmung für Nieren-Clearanceunstersuchungen bei Stoffwechselgesunden und Diabetikern. Klin Wochenschr 33:729-730

34. Vurek GG, Pegram SE 1966 Fluorometric method for determination of nanogram quantities of inulin. Anal Biochem 16:409-419

35. Bankir L, Bouby N, Trinh-Trang-Tan MM 1987 Heterogeneity of nephron anatomy. Kidney Int 31:S25-S39

36. Oliver J 1968 Nephrons and Kidneys: A Quantitative Study of Developmental and Evolutionary Mammalian Renal Architectonics. Hoeber Medical Division, Harper and Row, New York

37. Aschinberg LC, Koskimies O, Bernstein J, Nash M, Edelman Jr CM, Spitzer 
A 1978 The influence of age on the response to renal parenchymal loss. Yale J Biol Med 51:341-345

38. Ichikawa I, Yoshida Y, Fogo A, Purkerson ML, Klahr S 1988 Effect of heparin administration on the glomerular structure and function of remnant nephrons. Kidney Int 34:638-644

39. Yoshida Y, Kawamura T, Ikoma M, Fogo A, Ichikawa I 1989 Effect of antihypertensive drugs on glomerular morphology. Kidney Int 36:626-635

40. Kasiske BL, O'Donnell MP, Cleary MP, Keane WF 1988 Treatment of hyperlipidemia reduces glomerular injury in obese Zucker rats. Kidney Int 33:667-672

41. Zatz R, Dunn BR, Meyer TW, Anderson S, Rennke HG, Brenner BM 1986 Prevention of diabetic glomerulopathy by pharmacological amelioration of glomerular hypertension. J Clin Invest 77:1925-1930
42. Bank N, Klose R, Aynedjian HS, Nguten D, Sablay LP 1987 Evidence against increased glomerular pressure initiating diabetic nephropathy. Kidney Int 31:898-905

43. Yoshida Y, Fogo A, Glick A, Ichikawa I 1988 Serial micropuncture analysis of single nephron function in the rat model of subtotal ablation. Kidney Int 33:855-867

44. Fogo A, Yoshida Y, Glick AD, Homma T, Ichikawa I 1988 Serial micropuncture analysis of glomerular function in two rat models of glomerular sclerosis. J Clin Invest 82:322-330

45. Dworkin DL, Parker M, Feiner HD 1989 Nifedipine decreases glomerular injury in rats with remnant kidneys by inhibiting glomerular hypertrophy. Kidney Int 35:427(abstr) 